Karlik, E., et al., Transposon Studies on Colchium chalcedonicum. International Journal of Life Sciences and Biotechnology, 2019. 2(1): p. 25-35.

\title{
Transposon Studies on Colchium chalcedonicum
}

\author{
Elif Karlik*1, 2, Merve Albayrak ${ }^{3}$, Erdal Uzen ${ }^{4}$, Nermin Gozukirmizi ${ }^{2,5}$
}

\begin{abstract}
Colchicum chalcedonicum is one of the endemic plants in Turkey. The aim of this study was the investigation of the retrotransposon SIRE1, Sukkula and Nikita presence and insertion patterns in $C$. chalcedonicum. The plant samples were collected from the botanic garden of the Istanbul University. DNA isolation was performed from leaves by using modified CTAB/SEVAG protocol. Retrotransposon movements were investigated using SIRE1, Sukkula and Nikita primers by Inter Retrotranposon Amplified Polymorphism PCR technique (IRAP-PCR). Polymorphism percentages (\%) were calculated based on Jaccard Similarity Index. We observed that polymorphism ratios of SIRE1, Sukkula and Nikita retrotransposons among all samples were 0-40\%, 0$100 \%$ and $0-60 \%$, respectively. This is the first report to demonstrate three barley SIRE1, Sukkula and Nikita- retrotransposons presence and movements in $C$. chalcedonicum which is belonged to Colchium family, thus these IRAP primers may be used in further characterization and diversity studies of Colchicum family.
\end{abstract}

\author{
ARTICLE HISTORY \\ Received \\ 19 February 2019 \\ Accepted \\ 26 March 2019
}

\section{KEYWORDS}

Colchicum

chalcedonicum,

Retrotransposons,

SIRE1, Sukkula, Nikita

\section{Introduction}

Ecology of Turkey is very favourable for rich flora, because of its geographical location, topographic features, environmental and climate convenience at the cross section of three phytogeographic regions. Approximately 900 geophyte taxa (bulbs, tubers and rhizomes plants) grow naturally in Turkey [1]. Geophytes mostly consist of Araceae, Liliaceae, Primulaceae, Iridaceae, Geraniaceae, Orchidaceae, Ranunculaceae, Amaryllidaceae families that some of them have medical and economical properties [2-4]. Colchicum family, which is belonged to Liliaceae, are presented as 39 species in Turkey that one of the endemic species of Colchicum is Colchicum chalcedonicum which is native to Asia, Europe and Africa. C. chalcedonicum, which is also called as Kadikoy (Chalcedon) crocus, was first collected by Aznavour [5]. C.

\footnotetext{
${ }^{1}$ The University of Istanbul, Institution of Graduate Studies in Science and Engineering, Department of Biotechnology, Istanbul/Turkey

${ }^{2}$ The University of Istinye, Faculty of Arts and Sciences, Department of Molecular Biology and Genetics, Istanbul/Turkey,

${ }^{3}$ The University of Gebze Technical University, Institution of Natural and Applied Sciences, Department of

Molecular Biology and Genetics, Kocaeli/Turkey

${ }^{4}$ The University of Istanbul, Faculty of Science, Department of Biology, Istanbul/Turkey

${ }^{5}$ The University of Istanbul, Faculty of Science, Department of Molecular Biology and Genetics, Istanbul/Turkey

*Corresponding author: Elif Karlık, elif.karlik@istinye.edu.tr
} 
chalcedonicum has usually 4 leaves, long-oval shapes corms under the soil, its chromosome number is $2 \mathrm{n}=50$, and grows dry stones and rocky places [6-8].

Eukaryotic genomes comprise an abundance of repeated DNA which can move from one location of the genome to another, are also defined as transposable elements (TEs) can move within or between genomes [9]. More than 50 years ago, TEs were first identified by geneticist Barbara McClintock [10]. Nowadays, it is known that transposons are found in almost all organisms. Different types of TEs have been described that important difference between TE types is the presence of the reverse transcriptase (i.e. the transcription of RNA into DNA). Therefore, TEs are categorized into two groups based on their transposition mechanism and structural features: the retrotransposons (class I) and the DNA transposons (class II) [9].

Plants mostly contain retrotransposons more than $80 \%$ of in their genomes such as maize, wheat and barley [11, 12]. Retrotransposons use RNA to move new chromosomal locations that this mechanism is also called as "copy and paste" mechanism. Additionally, retrotransposons are subdivided into two groups; (1) long terminal repeats (LTR) retrotransposons, and (2) non-LTR retrotransposons. These repeats have a role in the insertion of the TEs which are also defined as "footprints" when the TEs are excised [13]. During speciation and evolution, TEs enlarged a large percentage of genome volume as demonstrated in plants [14], Drosophila or primates [15-17]. Due to the transposition event such as insertions, excisions, duplications or translocations, TEs can produce genetic variations [18-22]. Some studies showed that DNA transposons can alter the expression by insertion of specific regions in the genome such as introns, exons or regulatory elements. Moreover, TEs can be reorganized the genome by the mobilization of non-transposon DNA. In addition, TEs act as recombination substrates trigger recombination between two sequences of a transposon placed in the same or different chromosomes, which could be the origin for several types of chromosome alterations. Hence, TEs can be resulted in the loss of genomic DNA by internal deletions [23-25].

SIRE1 is plant specific LTR retrotransposon belonging to the sirevirus class of the Ty1-Copia retrotransposon family have their own genome structure among LTR retrotransposons according to possessing a putative envelope-like (ENV-like) gene immediately downstream of the reverse transcriptase gene [13]. Each copy of SIRE1 is appx. $11 \mathrm{~kb}$, making SIRE1 one of the largest retroelement in soybean, additionally, SIRE1 is active in the other plant species such as barley [26, 27]. Another LTR retrotransposon, which was first identified in barley by Shirasu, is Sukkula retroelement is approximately $5 \mathrm{~kb}$, containing reverse transcriptase in appx. $3.5 \mathrm{~kb}$ central domain which is found to be conserved as in primary sequence and secondary structure. 
However, Sukkula includes no open reading frames (ORFs) encoding typical retroelement proteins. According to these features of Sukkula, a novel group of retrotransposons, large retrotransposon derivatives or LARDs, have been described that they are member of the gypsy class of LTR retrotransposons, are similar to TRIMs (Terminal-repeat Retrotranposons in Miniature) in their lack of a protein-coding domain [28]. Nikita was the $4^{\text {th }}$ TE reported in barley has been used to determine polymorphism in polyploids, genetic variability, comparison of different retrotransposon-based marker techniques and hybrids [29-33]. For genome diversification in plants, active retrotransposons are mostly considered as major contributors because of their transposition and accumulation potentials in the genome [34-36].

Molecular marker techniques have become an important tool in molecular plant breeding [37]. Inter Retrotranposon Amplified Polymorphism (IRAP) is a molecular marker technique based on retrotransposon movements. Currently IRAP molecular markers are widely used to investigate polymorphism among individuals, species identification and environmental effects on individual's genetic diversity in plants. Retrotransposons integrate a daughter copy, which causes new joints between genomic DNA and the conserved LTRs; therefore, they can be used as markers. IRAP PCR neither requires restriction enzyme digestion or ligation to produce the marker bands is based on polymerase chain reaction amplification [38]. IRAP PCR technique determines retrotransposon insertional polymorphisms by amplifying the portion of DNA between two retroelements. One or two primers pointing outwards from an LTR are used, thus IRAP PCR amplifies the tract of DNA between two nearby retrotransposons. The result of IRAP PCR products are called as 'the fingerprints', from amplification of hundreds to thousands of target sites in the genome [37,38].

The aim of this study was to identify well-studied plant LTR-retrotransposons, including SIRE1, Sukkula and Nikita in C. chalcedonicum. For this purpose, SIRE1, Sukkula and Nikita insertion patterns were investigated using IRAP analysis method and results were analysed by Jaccard Similarity Index technique. This is the first report to demonstrate SIRE1, Sukkula and Nikita retrotransposons insertion patterns in C. chalcedonicum.

\section{Materials and Methods}

\section{Plant material}

C. chalcedonicum leaves used in this study were kindly provided from Erdal Uzen and the specimen was deposited in the Botanic Garden of Istanbul University. 


\section{DNA extraction}

Genomic DNA extraction was performed with some modifications using CTAB/SEVAG protocol. A total amount of $100 \mathrm{mg}$ of each sample were homogenized by grinding in liquid nitrogen. For total DNA extraction, $100 \mathrm{mg}$ grounded samples were incubated with $500 \mathrm{ml}$ $\mathrm{CTAB}$ buffer solution at $65^{\circ} \mathrm{C}$ for $1 \mathrm{~h}$. Then, the samples were centrifuged for $15 \mathrm{~min}$ at 16,000 $\mathrm{x}$ g. Afterwards, the aqueous phase was transferred to fresh tubes by pipette, then $200 \mu \mathrm{l}$ poteinase $\mathrm{K}(25 \mathrm{mg} / \mathrm{ml})$ and $5 \mu \mathrm{RNase}$ A solution $(10 \mathrm{mg} / \mathrm{ml})$ were added and incubated at 65 ${ }^{\circ} \mathrm{C}$ for $30 \mathrm{~min}$. Next, the samples were centrifuged for $10 \mathrm{~min}$ at $16,000 \mathrm{x} \mathrm{g}$. The supernatants were transferred to fresh tubes including equal volume of SEVAG (phenol:chloroform:isoamyl alcohol, 25:24:1), the samples were mixed and centrifuged for $10 \mathrm{~min}$ at $16000 \mathrm{x} \mathrm{g.} \sim 500 \mathrm{ml}$ of aqueous phase was transferred to fresh tubes which consisted of equal volume of chloroform. Afterwards, the samples were centrifuged for $10 \mathrm{~min}$ at $16000 \mathrm{x} \mathrm{g}$. The aqueous phase of samples was mixed with 2 volumes of CTAB precipitation solution and incubated at room temperature for an hour. Following centrifugation was carried out for $5 \mathrm{~min}$ at $16000 \mathrm{x} \mathrm{g}$. The pellet of the samples was dissolved in $350 \mathrm{ml} 1.2 \mathrm{M} \mathrm{NaCl}$ solution, then $350 \mathrm{ml}$ chloroform were added and centrifuged for $10 \mathrm{~min}$ at $16000 \mathrm{x}$ g. The aqueous phase was transferred to tubes containing 0.6 volumes of isopropanol and incubated at $-20^{\circ} \mathrm{C}$ for $30 \mathrm{~min}$. The samples were centrifuged for $10 \mathrm{~min}$ at $16000 \mathrm{x}$ g. Afterwards, the pellets were washed with $70 \%$ ethanol and dissolved in $30 \mathrm{ml}$ of sterile $\mathrm{dH}_{2} \mathrm{O}$. Degradation of the isolated DNA was assessed by using $1 \%$ agarose gel electrophoresis. Spectrophotometric analysis of the DNA quantity and quality were measured at 230, 260 and 280nm by NanoDrop 2000c UV-Vis spectrophotometer (Thermo Scientific, 2000c) For IRAP analysis, the DNA's concentrations were equalized to 20 $\mathrm{ng} / \mu \mathrm{L}$.

\section{IRAP PCR analysis}

SIRE1, Sukkula and Nikita insertion patterns were investigated using modified IRAP-analysis. The primer sequences used in this study was shown in Table 1. SIRE1, Sukkula and Nikita IRAP-PCR analysis were carried out using a thermal cycler in a total volume of $20 \mu \mathrm{L}$, containing $4 \mu \mathrm{L}$ of sterile $\mathrm{dH}_{2} \mathrm{O}, 2 \mu \mathrm{L}$ of primer $(1 \mu \mathrm{M} / \mu \mathrm{L}), 4 \mu \mathrm{L}$ of $20 \mathrm{ng} / \mu \mathrm{L}$ template genomic DNA (80 ng/ $\mu \mathrm{l})$ and $10 \mu \mathrm{L}$ of $2 \mathrm{X}$ Illustra ${ }^{\mathrm{TM}}$ Hot Start Master Mix (GE Healthcare, Sigma). The values given in parentheses were the final concentrations. PCR conditions were as follows: initial denaturation at $94^{\circ} \mathrm{C}(3 \mathrm{~min})$, followed by 40 cycles of denaturation at $94^{\circ} \mathrm{C}(30 \mathrm{~s})$, annealing at $57^{\circ} \mathrm{C}$ for SIRE 1 , at $55^{\circ} \mathrm{C}$ for Sukkula and at $51^{\circ} \mathrm{C}$ for Nikita $(30 \mathrm{~s})$ and extension at $72^{\circ} \mathrm{C}(3 \mathrm{~min})$. The reaction was completed by additional extension at $72^{\circ} \mathrm{C}$ for $10 \mathrm{~min}$. 
Table 1 List of primers used in this study.

\begin{tabular}{lllc}
\hline No & Primer Name & Sequence $\left(\mathbf{5}^{\prime} \rightarrow \mathbf{3}^{\prime}\right)$ & References \\
\hline 1 & SIRE1 & GCAGTTATGCAAGTGGGATCAGC & {$[38]$} \\
\hline 2 & Nikita & CGCATTTGTTCAAGCCTAAACC & {$[40]$} \\
\hline 3 & Bagy2 F & CTCGCTCGCCCACTACATCAACCGCGTTTATT & {$[41]$} \\
\hline 4 & Bagy2 R & ATCATTCCCTCTAGGGCATAATTC & {$[41]$} \\
\hline
\end{tabular}

\section{Evaluation of polymorphism results}

The percentages of polymorphism (\%) were calculated using Jaccard similarity index [42]. In brief, bands result of IRAP PCR were scored as a binary value: ' 1 ' for presence and ' 0 ' for absence; the binary matrix $(1 / 0)$ was then utilized to calculate the similarity between the different individuals using Jaccard's similarity index. Moreover, band profiles were also analysed by GelJ v.2.0 to construct the phylogenetic tree, UPGMA (unweighted pair-group method with arithmetic mean) clustering method with Jaccard's Similarity Index was utilized to cluster the subjects according to band distances on gel images [43].

\section{Results}

Retrotransposons -SIRE1, Sukkula and Nikita- movements in C. chalcedonicum have been analysed using IRAP-PCR technique. IRAP-PCR results were electrophoretically separated in a 2\% agarose gel (see Fig 1). SIRE1 and Sukkula IRAP band profiles were ranged from 100 to $400 \mathrm{bp}$. However, IRAP band profiles of Nikita were ranged from to 300 to $2000 \mathrm{bp}$.

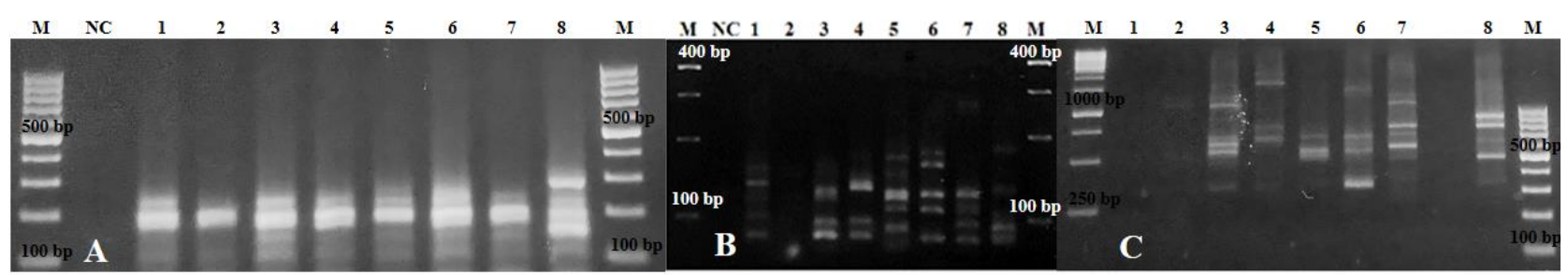

Fig 1 Demonstration of IRAP PCR products separated in 2\% agarose gel. A: SIRE1 results. B: Sukkula results. C: Nikita results. M: 100 bp marker and 1 kb marker. NC: no template control. 1-8: PCR products obtained from leaves of individual seedlings

The resulting IRAP-PCR amplification products demonstrated that polymorphism ratios of SIRE1, Sukkula and Nikita retrotransposons among all samples were 0-40\%, 0-100\% and 0$60 \%$, respectively (see Table 2 and 3). 
Table 2 Polymorphism percentages (\%) of SIRE1 and Sukkula. A: SIRE1; B: Sukkula

\begin{tabular}{l|lllllllllllllllll}
\hline \multicolumn{1}{l}{$\mathbf{A}$} & 1 & 2 & 3 & 4 & 5 & 6 & 7 & 8 & $\mathbf{B}$ & 1 & 2 & 3 & 4 & 5 & 6 & 7 & 8 \\
\hline 1 & - & & & & & & & & 1 & - & & & & & & & \\
2 & 25 & - & & & & & & & 2 & $\mathbf{1 0 0}$ & - & & & & & \\
3 & 17 & 0 & - & & & & & & 3 & 10 & 10 & - & & & & \\
4 & 0 & 0 & 0 & - & & & & & 4 & 22 & 22 & 11 & - & & & \\
5 & 0 & 0 & 0 & 17 & - & & & & 5 & 0 & 0 & 10 & 22 & - & & & \\
6 & 0 & 20 & 0 & $\mathbf{4 0}$ & 14 & - & & & 6 & 0 & 0 & 33 & 9 & 8 & - & & \\
7 & 0 & 0 & 0 & 17 & 0 & 14 & - & & 7 & 0 & 0 & 9 & 9 & 18 & 17 & - & \\
8 & 14 & 0 & 29 & 0 & 0 & 0 & 0 & - & 8 & 9 & 9 & 0 & 22 & 20 & 8 & 18 & - \\
\hline
\end{tabular}

Table 3 Polymorphism percentages (\%) of Nikita

\begin{tabular}{l|llllll}
\hline $\mathbf{C}$ & 3 & 4 & 5 & 6 & 7 & 8 \\
\hline 3 & - & & & & & \\
4 & 25 & - & & & & \\
5 & 14 & 0 & - & & & \\
6 & 25 & 0 & $\mathbf{6 0}$ & - & & \\
7 & 0 & 10 & 0 & 0 & - & \\
8 & 9 & 0 & 0 & 0 & 8 & - \\
\hline
\end{tabular}

As a result of GelJ analysis, two main clusters have been observed for all retrotransposons -SIRE1, Sukkula and Nikita- used in this study. For SIRE1 clustering based on UPGMA dendrogram, first group consisted of 4 subjects (No: 3, 4, 5 and 10), and second group contained the other four -6, 7, 8 and 9- subjects. However, first cluster of Sukkula only comprised two subjects 3 and 4; the other 6 subjects were found in the second cluster. Additionally, clustering of Nikita was only resulted in 6 subjects thus, first cluster contained 4 subjects (No: 4, 5, 6 and 7 ) and the $2^{\text {nd }}$ group consisted of 2 subjects (No: 8 and 10) (see Fig 2). 


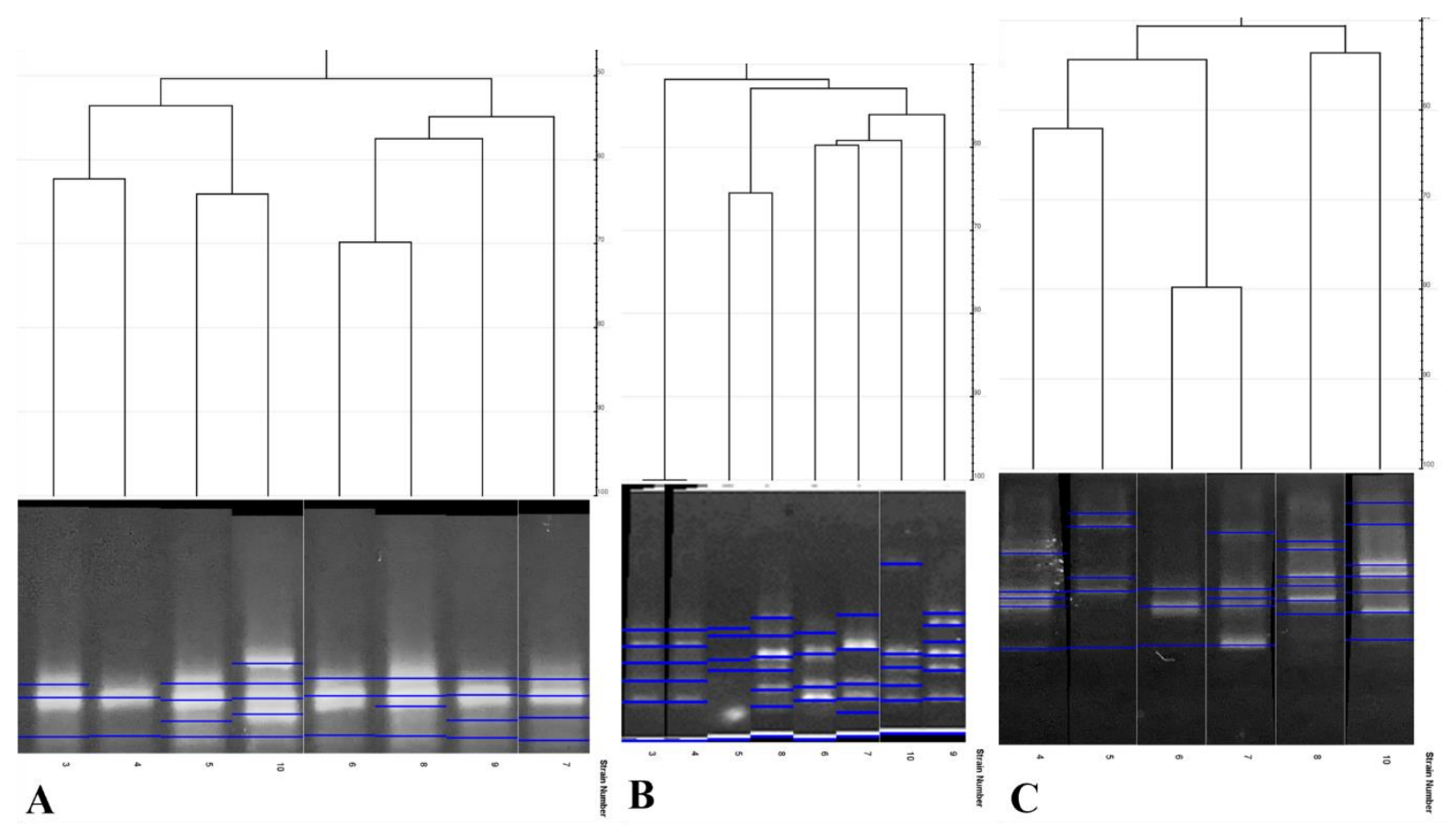

Fig 2 Clustering of subjects based on IRAP PCR amplification using SIRE1, Sukkula and Nikita specific primers (GelJ v.2.0 analysis). A: SIRE1 clustering. B: Sukkula clustering. C: Nikita clustering. 1-8: PCR products obtained from leaves of individual seedlings

\section{Discussion}

Transposable elements are widespread and dynamic elements of the genome, especially, they consist of the $80 \%$ of the genome in cereals [44]. According to their transposition mechanism, TEs can alter gene products and gene expression profiles [45]. In addition, TEs have considered as an important motive for genome evolution and speciation due to their dynamic feature [30]. Retrotransposons are usually utilized to determine genetic relationships between varieties and related species based on their variation capacity between species [46-48]. The aim of this study was to determine the presence and insertion patterns of the SIRE1, Sukkula and Nikita retrotransposons in C. calcedonicum. Polymorphism percentages (\%) were calculated as 0$40 \%, 0-100 \%$ and $0-60 \%$ for SIRE1, Sukkula and Nikita, respectively, according to IRAP PCR results due to Jaccard Similarity Index. Two main clusters have been observed for all three retrotransposon types. - SIRE1, Sukkula and Nikita-

Several plant species, which are widespread and endemic, are placed in Anatolia is the richest regions in Turkey [49]. Moreover, Turkey has the extensive distribution of Colchicum family consist of 39 species that 15 of them endemic [50-52]. However, chromosome number was only reported associated with $C$. chalcedonicum genome. Our study is the first report demonstrating 
TE presence and insertion patterns in C. chalcedonicum genome. TEs are widespread in the plant kingdom, thus they participate of several common features, both structural and mechanistic, with mobile elements from other eukaryotes [53]. We observed that IRAP primers of SIRE1, Sukkula and Nikita retrotransposons were studied in C. chalcedonicum genome, indicating that these retrotransposons were presence and active in C. chalcedonicum. Moreover, genetically active TEs could be distinguished through genome [54, 55].

Numerous studies have reported the number, diversity, and distribution of transposable elements within plant genomes [56-59]. SIRE1, which is a family of copia/Ty1-related retrotransposons in the soybean genome, is most closely related to opie-2 from maize. Both Glycine max and Glycine soja comprise of SIRE1 elements, suggesting this retrotransposon family was present before soybean domestication [60]. However, our studies demonstrated that SIRE1 is still active in soybean, indicating this family may proceed to be proliferated in the soybean genome (unpublished data). SIRE1 retrotransposon family was also studied in barley and human genomes by Cakmak et al. [27; unpublished data]. In barley, Cakmak et al. [27] have found polymorphism rates between $0-64 \%$ among all samples. Also, polymorphism percentages (\%) were observed as $0-40 \%$ in our study for SIRE1 IRAP primer. Our results indicated that SIRE1 IRAP primer is suitable for further characterization and diversity studies.

Several studies have been performed for genetic characterization and diversity analysis using Sukkula retrotransposon [61-63]. However, Sukkula, which means "shuttle" in Finnish, have been reported the $2^{\text {nd }}$ most active retrotransposon in the barley genome [29]. Moreover, Sukkula have been proposed to be associated with intraspecies variations by Kartal-Alacam et al. [63], which demonstrated the polymorphism rates up to $61 \%$ and $70 \%$ using IRAP, and iPBS, respectively. Moreover, Sukkula movements have been observed in human that polymorphism percentages (\%) were found to be $8-100 \%$ among all samples; $10-91 \%$ in 12 female subjects and $13-100 \%$ in 12 male subjects [64]. In our study, we observed 0-100\% polymorphism rate, suggesting that Sukkula retrotransposon is favourable to perform Colchicum characterization and diversity studies.

Although Nikita retrotransposon have been reported as the fourth most active retrotransposon in barley [29], studies associated with Nikita is quite limited. Bayram et al. [65] have only observed some polymorphic bands ( 550 and $650 \mathrm{bp})$ in callus culture, indicating that tissue culture conditions may be responsible for the movement of the Nikita. Additionally, Sukkula and Nikita IRAP primer transferability have been demonstrated in Pimpinella anisum L. by Marakli [66]. Interestingly, polymorphism rate of Nikita in this study was calculated as 0-60\%, 
suggesting that Nikita IRAP primer may also be used in further characterization and diversity studies.

\section{Conclusion}

C. calcedonicum is an endemic and valuable plant for Turkey. Comparison of three IRAP primers - SIRE1, Sukkula and Nikita- used in this study demonstrated that Sukkula IRAP primer is favourable for Colchicum family according to polymorphism range of molecular marker technique.

\section{Acknowledgments}

This study was supported by Kadıköy Municipality. Also, the authors thank Meltem Deger for sharing results and studies on Colchicum species.

\section{References}

1. Koyuncu, M. and Ş. Alp, New geophyte taxa described from Turkey at last decade. Yüzüncü Yıl University Journal of Agriculture Science, 2014. 24 (1): p. 101-110.

2. Baytop, T., Therapy with plant in Turkey, İstanbul University Publication. 1984, Istanbul.

3. Sargın, A.S., S. Selvi, and E. Akçiçek, Investigations of ethnobotanical aspect of some geophytes growing in Alaşehir (Manisa) and surrounding area, Erciyes University Journal of Institute of Science and Technology, 2013. 29(2): p. 170-177.

4. Atak, A., E. Kaya, and K. Erken, Determination of quantity and purity of some geophytes collected from the flora of Turkey, Asian Journal of Plant Sciences, 2014. 13(3): p. 98-110.

5. Aznavour, G.Y., Note sur la flore des environs de Constantinople. Bulletin de la Société Botanique de France, 1987. 44: p. 164-177.

6. Brickell, C.D., Colchicum L, In: Davis PH (ed.), Flora of Turkey and the East Aegean Islands. Vol. 8, University Press, 1984. Edinburgh.

7. Küçüker, O., The morphological, anatomical and cytological studies on some Colchicum species of Istanbul area. Journal of Science Istanbul University, 1985. 50(B): p. 87-111.

8. Persson, K., Nomenclatural synopsis of the genus Colchicum (Colchicaceae), with some new species and combinations. Botanische Jahrbücher für Systematik, 2007. 127: p. 165-242.

9. Pray, L., Transposons: The jumping genes. Nature Education, 2008. 1(1): p. 204.

10. McClintock, B., The origin and behavior of mutable loci in maize. Proceedings of the National Academy of Sciences of the United States of America, 1950. 36(6): p. 344-355.

11. Liu, R., et al., A GeneTrek analysis of the maize genome. Proceedings of the National Academy of Sciences of the United States of America, 2007. 104: p. 11844-11849.

12. Wicker, T., et al., A whole-genome snapshot of 454 sequences exposes the composition of the barley genome and provides evidence for parallel evolution of genome size in wheat and barley. Plant Journal, 2009. 59: p. 712-722.

13. Gao, X., et al., Translational recoding signals between gag and pol in diverse LTR retrotransposons. RNA, 2003. 9(12): p. 1422-1430.

14. SanMiguel, P., et al., The paleontology of intergene retrotransposons of maize. Nature Genetics, 1998. 20(1): p. 43-45.

15. Sheen, F.M., and R.W., Levis, Transposition of the LINE-like retrotransposon TART to Drosophila chromosome termini. Proceedings of the National Academy of Sciences of the United States of America, 1994. 91: p. 12510-12514. 
16. Frazer, K.A., et al., Genomic DNA insertions and deletions occur frequently between humans and nonhuman primates. Genome Research, 2003. 13(3): p. 341-346.

17. Locke, D.P., et al., Large-scale variation among human and great ape genomes determined by array comparative genomic hybridization. Genome Research, 2003. 13(3): p. 347-357.

18. Jordan, E., H. Saedler, and P. Starlinger, $\mathrm{O}^{0}$ and strong-polar mutations in the gal operon are insertions. Molecular and General Genetics, 1968. 102(4): p. 353-363.

19. Rubin, G.M., M.G. Kidwell, and P.M. Bingham, The molecular basis of P-M hybrid dysgenesis: the nature of induced mutations. Cell, 1982. 29(3): p. 987-994.

20. Kazazian, H.H., et al., Haemophilia a resulting from de novo insertion of L1 sequences represents a novel mechanism for mutation in man. Nature, 1988. 332(6160): p. 164-166.

21. Clegg, M.T, and M.L. Durbin, Tracing floral adaptations from ecology to molecules. Nature Reviews Genetics, 2003. 4(3): p. 206-215.

22. Lerman, D.N, and M.E. Feder, Naturally occurring transposable elements disrupt hsp70 promoter function in Drosophila melanogaster. Molecular Biology and Evolution, 2005. 22(3): p. 776-783.

23. Kidwell, M.G, and D.R. Lisch, Perspective: transposable elements, parasitic DNA, and genome evolution. Evolution, 2001. 55(1): p. 1-24.

24. Kazazian, H.H., Mobile elements: drivers of genome evolution. Science, 2004. 303(5664), p. $1626-1632$.

25. Feschotte, C, and E.J. Pritham, DNA transposons and the evolution of eukaryotic genomes. Annual Review of Genetics, 2007. 41: p. 331-368.

26. Laten, H.M, and R.O. Morris, SIRE1, a long interspersed repetitive DNA element from soybean with weak sequence similarity to retrotransposons: initial characterization and partial sequence. Gene, 1993. 134(2): p. 153-159.

27. Çakmak, B., S. Marakli, and N. Gözükirmizi, SIRE1 Retrotransposons in Barley (Hordeum vulgare L.). Russian Journal of Genetics, 2015. 51(7): p.661-672.

28. Kalendar, R., et al., Large Retrotransposon Derivatives: Abundant, Conserved but Nonautonomous Retroelements of Barley and Related Genomes. Genetics, 2004. 166: p.1437-1450.

29. Leigh, F., et al., Comparison of the utility of barley retrotransposon families for genetic analysis by molecular marker techniques. Molecular Genetics and Genomics, 2003. 269: p. 464-474.

30. Bento, M., et al., Polyploidization as a retractionforce in plant genome evolution: sequence rearrangements in Triticale. PLoS One, 2008. 3: p. e1402.

31. Bento, M., et al., Genome merger: from sequence rearrangements in Triticale to their elimination in wheat-rye addition lines. Theoretical and Applied Genetics, 2010. 121: p. 489-497.

32. Carvalho, A., et al., Genetic variability of Old Portuguese bread wheat cultivars assayed by IRAP and REMAP markers. Annals of Applied Biology, 2010. 156: p. 337-345.

33. Patel, D., et al., Somatic hybrid plants of Nicotiana X sanderae (+) N. debneyi with fungal resistance to Peronospora tabacina. Annals of Botany-London, 2011. 108: p. 809-819.

34. Wessler, S.R., T.E. Bureau, and S.E. White, LTRretrotransposons and MITEs: important players in the evolution of plant genomes. Current Opinion in Genetics and Development, 1995. 5(6): p. 814-821.

35. Vicient, C.M., et al., Retrotransposon BARE-1 and Its Role in Genome Evolution in the Genus Hordeum. Plant Cell, 1999. 11: p. 1769-1784.

36. Schulman, A.H, and R. Kalendar, A movable feast: diverse retrotransposons and their contribution to barley genome Dynamics. Cytogenetic and Genome Research, 2005. 110: p. 598-605.

37. Kalendar, R, and A.H. Schulman, IRAP and REMAP for retrotransposon-based genotyping and fingerprinting. Nature Protocols, 2006. 1(5): p. 2478-2484.

38. Kalendar, R, and A.H. Schulman, Transposon based tagging: IRAP, REMAP, and iPBS. Methods in Molecular Biology, 2014. 1115: p. 233-255.

39. Chesnay, C., A. Kumar, and S.R. Pearce, Genetic diversity of SIRE1 retroelements in annual and parennial glycine species revealed using SSAP. Cellular and Molecular Biology Letters, 2007. 12: p. $103-$ 110.

40. Rodriguez, M., et al., Integration of Retrotransposons-Based Markers in a Linkage Map of Barley, Molecular Breeding, 2006. 17: p. 173-184.

41. Zein, I., M. Jawhar, and M.I.E. Arabi, Efficiencyof IRAP and ITS-RFLP marker systems in accessing genetic variation of Pyrenophora graminea. Genetics and Molecular Biology, 2010. 33(2): p. 328-332. 
42. Jaccard, P., Nouvelles recherches sur la distribution florale [New research on floral distrubition]. Bulletin de la Société Vaudoise des Sciences Naturelles, 1908. 44: p. 223-270.

43. Heras, J., et al., GelJ- a tool for analyzing DNA fingerprint gel images. BioMed Central Bioinformatics, 2015. 16: p. 270.

44. Mascher, M., et al., A chromosome conformation capture ordered sequence of the barley genome. Nature, 2017. 544: p. 427-433.

45. Fedoroff, N, Transposons and genome evolution in plants. Proceedings of the National Academy of Sciencies of the United States of America, 2000. 97(13): p. 7002-7007.

46. Baumel, A., et al., Retrotransposons and genomic stability in populations of the young allopolyploid species Spartina anglica C.E. Hubbard (Poaceae). Molecular Biology and Evolution, 2002. 19(8): p. 1218-1227.

47. Alavi-Kia, S.S., et al., Analysis of genetic diversity and phylogenetic relationships in Crocus genus of Iran using Inter Retrotransposon Amplified Polymorphism. Biotechnology and Biotechnological Equipment, 2008. 22: p. 795-800.

48. Belyayev, A., et al., Transposable elements in a marginal plant population: temporal fluctuations provide new insights into genome evolution of wild diploid wheat. Mobile DNA, 2010. 1: p. 1-16.

49. Giray, B, Türkiye'deki Doğal Çiçek Soğanları ile ilgili Gelişmeler. Tarım ve Köyişleri Bakanlığı Dergisi. 139. 2001. Ankara.

50. Dahlgren, R.M.T., H.T. Clifford, and P.F. Yeo, The Families of the Monocotyledons -Structure, Evolution and Taxonomy. 1985. Springer-Verlag Berlin Heidelberg: New York.

51. Persson, K, The Genus Colchicum in Turkey I. New Species. Edinburgh Journal of Botany, 1999. 56(1): p. $85-102$.

52. Akan, H, and I. Eker, Check-list of the genus Colchicum in the flora of Turkey. Turkish Journal of Botany, 2005. 29: p. 327-331.

53. Flavell, A.J., S.R. Pearce, and A. Kumar, Plant transposable elements and the genome. Current Opinion in Genetics and Development, 1994. 4(6): p. 838-844.

54. Fedoroff, N.V., D.B. Furtek, and O.E. Nelson Jr., (1984) Cloning of the bronze locus in maize by a simple generalizable procedure using the transposable controlling element Activator (Ac). Proceedings of the National Academy of Sciencies of the United States of America, 1984. 81: p. 3825-3829.

55. Banks, J.A, and N. Fedoroff, Patterns of developmental and heritable change in methylation of the Suppressor-mutator transposable element. Developmental Genetics, 1989. 10: p. 425-437.

56. Kumar, A, and J.L. Bennetzen, Plant retrotransposons. Annual Review of Genetics, 1999. 33: p. 479-532.

57. Feschotte, C., N. Jiang, and S.R. Wessler, Plant transposable elements: Where genetics meets genomics. Nature, 2002. 3: p. 329-341.

58. Deininger, P.L., et al., Mobile elements and mammalian genome evolution. Current Opinion in Genetics and Development, 2003. 13: p. 651-658.

59. Havecker, E.R., X. Gao, and D.F. Voytas, The diversity of LTR retrotransposons. Genome Biology, 2004. 5: p. 225.

60. Hymowitz, T, and C.A. Newell, Taxonomy of the genus Glycine: domestication and uses of soybeans. Economic Botany, 1981. 35: p. 272-288.

61. Carvalho, A., H. Guedes-Pinto, and J.E. Lima-Brito, Genetic diversity in old Portuguese durum wheat cultivars assessed by retrotransposon-based markers. Plant Molecular Biology Reporter, 2012. 30: p. 578-589.

62. Nasri, S., et al., Retrotransposon insertional polymorphism in Iranian bread wheat cultivars and breeding lines revealed by IRAP and REMAP markers. Biochemical Genetics, 2013. 51: p. 927-943.

63. Kartal-Alacam, G., et al., Sukkula retrotransposon insertion polymorphism in barley. Russian Journal of Plant Physiology, 2014. 61: p. 828-833.

64. Cakmak, B., S. Marakli, and N. Gozukirmizi, Sukkula retrotransposon movements in the human genome. Biotechnology and Biotechnological Equipment, 2017. 31(5): p. 900-905.

65. Bayram, E., et al., Nikita Retrotransposon Movements in Callus Cultures of Barley (Hordeum vulgare L.). Plantomics, 2012. 5: p. 211-215.

66. Marakli, S, Transferability of Barley Retrotransposons (Sukkula and Nikita) to Investigate Genetic Structure of Pimpinella anisum L. Marmara Fen Bilimleri Dergisi, 2018. 30: p. 299-304. 